\title{
A HEGEMONIA ESTADUNIDENSE E O PROTOCOLO ADICIONAL PARA APLICAÇÃO DE SALVAGUARDAS NO ÂMBITO DO TNP: UMA ANÁLISE A PARTIR DE GRAMSCI
}

\author{
Martha Kellen Gonçalves Machado ${ }^{1}$
}

\begin{abstract}
RESUMO
O Protocolo Adicional do Tratado de Não Proliferação foi criado para aumentar a aplicação de salvaguardas nos países não possuidores de armas nucleares. Os EUA têm se empenhado na defesa desse Protocolo, desde seu surgimento. O que desejam é que esse Protocolo entre em vigor em todos os países signatários, para dificultar a proliferação de armas nucleares. Essa posição dos EUA se submete a uma avaliação crítica pormenorizada, por meio do entendimento de hegemonia do pensador político Antonio Gramsci, aplicado às Relações Internacionais. Essa mesma hegemonia é vista no tratamento dispensado pelos EUA ao Protocolo Adicional, pois a Agência Internacional de Energia Atômica acatou esse Protocolo e tem feito o possível para que ele seja adotado unanimemente. Os EUA, por sua vez, têm agido de forma a mostrar os benefícios que os países que aceitarem esse Protocolo terão. Conclui-se que o conceito de hegemonia em Gramsci tem aplicabilidade eficaz na análise do Protocolo Adicional, e que a posição estadunidense se expressa como hegemônica.
\end{abstract}

Palavras-chave: Hegemonia; Gramsci; Protocolo Adicional do TNP; Não-Proliferação; Desarmamento.

\begin{abstract}
The Additional Protocol to the Non-Proliferation Treaty was created to increase the application of safeguards on non-nuclear states. The United States of America have pledged to support this Protocol since its establishment. The US aspires that the Protocol come into effect in all signatory states, in order to hinder the proliferation of nuclear weapons. The US position is thus submitted to a detailed critique through Antonio Gramsci's understanding of hegemony, by way of its use in International Relations. Hegemony is examined in the treatment the US has given to the Additional Protocol, since the International Atomic Energy Agency has accepted, and has done everything possible for its unanimous adoption. The US, in its turn, has acted in a way to demonstrate the benefits to be reaped should states accept the Protocol. The article concludes that the Gramscian concept of hegemony has an effective application in the analysis of the Additional Protocol, as well as in the expression of the US hegemonic position.
\end{abstract}

Key words: Hegemony; Gramsci; NPT Additional Protocol; Non-Proliferation; Disarmament.

\footnotetext{
${ }^{1}$ Aluna do curso de Relações Internacionais do UNICEUB.

AGRADEDIMENTOS: Primeiramente agradeço a Deus pela iluminação e pela paz de espírito. À minha família pelo apoio e pelo amor que sempre me deram. Ao meu querido esposo Fernando por ter me feito acreditar que era capaz de realizar este trabalho e ao meu professor Marco Antonio pela excelente orientação a mim dispensada.
} 


\section{Introdução}

Destina-se, este artigo, à abordagem de um dos mais importantes conceitos do pensador político Antonio Gramsci: a hegemonia. Essa abordagem será feita através da análise de uma realidade complexa das Relações Internacionais, de forma a enriquecê-la. A realidade a ser analisada será a estratégia e ação dos Estados Unidos na implementação internacional do Protocolo Adicional do Tratado de Não-Proliferação de Armas Nucleares (TNP), proposto pela Agência Internacional de Energia Atômica (AIEA), que visa a aumentar as salvaguardas dos países não nuclearmente armados. Não se tem a pretensão de promover nos estreitos limites deste trabalho uma análise minuciosa de um dos conceitos centrais da teoria política gramsciana, mas tão somente verificar se, à luz das idéias do pensador italiano, as posições adotadas pelos EUA, em relação ao Protocolo Adicional, conformam-se como medidas estrategicamente coerentes para a preservação da hegemonia norte-americana construída, em especial, ao longo do pós Segunda Guerra Mundial.

Desta forma, o artigo está estruturado em cinco partes. A primeira busca trazer uma revisão do conceito de hegemonia em Gramsci, bem como de alguns outros que lhe são intrinsecamente relacionados, buscando-se, desde já, mostrar sua aplicabilidade na apreciação das questões que incidem no cenário das Relações Internacionais. A segunda parte procura traçar, sinteticamente, a história norte-americana no segmento de armas nucleares, ressaltando algumas das suas principais ações para conter a proliferação de materiais nucleares. A terceira parte dedica-se a explicar, resumidamente, a origem e objetivos do Protocolo Adicional do TNP, e abordar de forma sintética as razões que levaram os EUA a defender veementemente o Protocolo Adicional, com o fim de deixar evidenciada a "renovação do velho", ou seja, a política de não-proliferação enquanto assegura seu poder de continuar possuindo armas nucleares. A quarta parte empenha-se a enfrentar a questão central proposta neste artigo, se a hegemonia norte-americana é a hegemonia de Gramsci, ou, em outras palavras, se à luz das idéias de Gramsci, a posição dos EUA frente ao Protocolo Adicional é uma ampliação de sua hegemonia. E, finalmente, uma quinta e última parte, a título de considerações finais.

\section{Hegemonia Gramsciana}

Quando esteve preso por perseguição política durante onze anos, o italiano Antonio Gramsci aproveitou os anos de reclusão, para escrever o que depois ficou conhecido como os "Cadernos do Cárcere"2. Neles é possível observar que "os conceitos de Gramsci foram todos derivados da história - a partir de suas próprias reflexões, em relação àqueles períodos os quais ele pensou terem lançado uma luz explanatória sobre o presente, e a partir de sua vivência pessoal de luta social e política"3. Diante disso, "uma importante particularidade das formulações conceituais propostas por Gramsci é a de que, são elas, em regra, flexíveis e abertas, só ganhando precisão quando conectadas às situações concretas

\footnotetext{
${ }^{2}$ Para maiores informações sobre a vida e escritos de Gramsci ver COUTINHO, Carlos Nelson. Gramsci: um estudo sobre seu pensamento politico. Rio de Janeiro: Civilização Brasileira, 1999. Gramsci não publicou nenhum livro durante sua vida. Toda a sua produção anterior à prisão é constituída por artigos, ensaios ou informes políticos. Os Cadernos do Cárcere nada mais são do que uma compilação de todos os seus escritos enquanto esteve preso.

${ }^{3}$ COX, Robert W. "Gramsci, Hegemony and International Relations: an essay in method" In GILL, Stephen. Gramsci, Historical Materialism and International relations. Cambridge University Press: 1993, p. 49. "Gramsci's concepts were all derived from history - both from his own reflections upon those periods of history which he thought helped to throw an explanatory light upon the present and from his personal experience of political and social struggle". Tradução própria.
} 
que buscam explicar. Não há conceitos fechados, mas noções que se remoldam continuamente" ${ }^{4}$. Ou seja, para compreender de forma profícua os conceitos propostos por este pensador político é necessário aplicá-los em alguma realidade ou fato.

A idéia de hegemonia do pensador italiano não foge à regra. Entre os renomados autores que se propuseram a entender os escritos de Gramsci, ainda não existe uma acepção estrita para o conceito. Ele é o resultado da conclusão de duas fontes de explanação: dos mecanismos de hegemonia da classe dominante na Europa Ocidental (burguesia), e dos escritos de Maquiavel.

Através dos mecanismos de hegemonia da classe dominante na Europa Ocidental (burguesia), Gramsci obteve a constatação de que o exercício do poder não demandava, exclusivamente, a detenção do aparato administrativo, executivo e coercitivo do governo. Devido à sua concepção de hegemonia estar firmemente entrincheirada na sociedade civil, a burguesia não precisava, ela própria, assumir o comando do Estado. Em verdade, a condição hegemônica era alcançada por via da atuação de vários segmentos da sociedade civil: a igreja, o sistema educacional, a imprensa, todas as instituições que ajudavam a criar nas pessoas certos modos de comportamento com a ordem social hegemônica. Esta percepção de hegemonia teve grande impacto em Gramsci, e serviu para que o pensador ampliasse sua definição de Estado ${ }^{5}$.

A outra fonte que leva para a idéia gramsciana de hegemonia vem dos escritos de Maquiavel e ajuda a alargar o escopo potencial da aplicação desse conceito. Gramsci extraiu de Maquiavel "a imagem de poder como um centauro: metade homem, metade animal, uma combinação necessária de consentimento e coerção"6, para explicar a hegemonia. Apesar de este conceito defender que o consentimento deva prevalecer em todas as esferas, a coerção não deve ser descartada; em sua visão ela deve ser latente, ou seja, existe potencialmente, mas não está presente. Como Mezzaroba exemplifica: “(...) um Estado só conquista efetivamente a hegemonia na medida em que não recorre a qualquer tipo de guerra ou violência, os fins devem ser alcançados através dos mecanismos da diplomacia, da ideologia, da economia, do conhecimento" ". Contudo a "força militar" deve ser calculada para observar a relação de poder entre os Estados ${ }^{8}$. Desta forma, Gramsci liberta o conceito de poder de uma vinculação estrita a um dado período histórico ou a certa classe social, dando-lhe uma aplicação mais ampla no âmbito das relações de dominação e subordinação, aí incluída as relações internacionais.

Para Coutinho, "a hegemonia é, sem lugar a dúvidas, o momento do consenso, (...) onde teria sua base material na sociedade civil, nos aparelhos privados de hegemonia"9. Ela não significa dominância, pelo contrário, é o oposto disso. É o consenso "no sentido de conduzir, de guiar" ${ }^{10}$. É um complexo de atividades culturais e ideológicas que organiza o consenso e permite o desenvolvimento da direção moderada. E o consenso é visto como "direção intelectual e moral". Sendo assim, o alcance da condição dirigente é pré-condição

\footnotetext{
${ }^{4}$ MICHELS, Gilson Wessler. "Guerra do Iraque e Hegemonia norte-americana: Uma leitura a partir de Gramsci" In MEZZAROBA, Orides (org.). Gramsci: Estado e Relações Internacionais. Florianópolis: Fundação Boiteux, 2005, p. 123.

${ }^{5}$ COX, Robert W. op. cit, p. 51.

${ }^{6}$ Idem, p. 52. "The image of power as a centaur: half man, half beast, a necessary combination of consent and coercion". Tradução própria.

7 MEZZAROBA, Orides. "Gramsci e a Hegemonia" In MEZZAROBA, Orides (org). Gramsci: Estado e Relações Internacionais. Florianópolis: Fundação Boiteux, 2005, p. 19.

${ }^{8}$ Idem, p. 18-19.

9 COUTINHO, Carlos Nelson. Gramsci: um estudo sobre seu pensamento político. Rio de Janeiro: Civilização Brasileira, 1999, p. 249.

${ }^{10}$ MEZZAROBA, Orides. Op. Cit, p. 9.
} 
para a conquista do poder. "Só se pode ser classe dominante quando já se é classe dirigente, quando já se detém o consenso da maioria da população trabalhadora"11.

Quando Gramsci observou que os mecanismos de hegemonia estavam dentro da sociedade civil, ele viu a necessidade de ampliar o conceito de Estado advindo de Marx. Esse Estado ampliado foi chamado de "Estado Integral", que é uma junção da sociedade civil com o Estado (que na idéia de Marx é a sociedade política). Como agora a sociedade civil faz parte da superestrutura, é preciso estabelecer, em seu âmbito, um sistema de alianças, de transformar seus interesses particulares em universais ou gerais, ou, em outras palavras, de fazer outro grupo encamparem seu conjunto de valores, para que dessa forma alcancem a hegemonia. Essa "posição ideológica que um país ocupa no mundo em cada momento determinado, enquanto considerado representante das forças progressistas da história" ${ }^{2}$, é um fator determinante para analisar a noção de hegemonia gramsciana.

Apesar de Gramsci ter se reservado em analisar as relações internas da Itália é possível verificar que suas idéias são amplas o suficiente para transportá-las para o campo das relações internacionais. Cox, refletindo sobre estas idéias, afirma que as inovações orgânicas - aquelas de longo prazo - dentro das relações sociais, modificam as relações internacionais, as quais possuem um fundo social, proveniente das alterações ocorridas no espaço interno dos Estados. "As relações internacionais antecedem ou seguem (logicamente) relações sociais fundamentais? Não há nenhuma dúvida de que elas seguem. Qualquer inovação orgânica na estrutura social, através das expressões técnicas-militares, modifica organicamente relações absolutas e relativas também no campo internacional"13. ${ }^{13}$ o símbolo do poderio de um país é observado pelas revoluções que ele tem passado, "aqueles Estados que são poderosos, são exatamente aqueles que têm passado por uma profunda revolução social e política e trabalharam o mais completamente as consequiências dessa revolução na forma de Estado e relações sociais" ${ }^{14}$.

No âmbito das Relações Internacionais, a hegemonia não está associada a uma ordem composta apenas por Estados. Como observa Robert Cox, "hegemonia é um complexo de relações sociais internacionais que conecta as classes sociais dos diferentes países. Ela é descrita como uma estrutura social, econômica e política; e não pode ser apenas uma dessas coisas, tem de ser todas elas. Além disso, ela é expressa em normas universais, instituições e mecanismos que podem estipular regras gerais de comportamento para os Estados e para as forças da sociedade civil que agem através das fronteiras nacionais" ${ }^{15}$.

\footnotetext{
${ }^{11}$ COUTINHO, Op. Cit, p. 69.

${ }^{12}$ MEZZAROBA, Orides. Op. Cit, p. 18-19.

${ }^{13}$ GRAMSCI, 1971: 176 Apud COX, Robert W. Gramsci, Hegemony and International Relations: an essay in method. In GILL, Stephen. Gramsci, Historical Materialism and International relations. Cambridge University Press: 1993, p 58. Do international relations precede or follow (logically) fundamental social relations? There can be no doubt that they follow. Any organic innovation in the social structure, through its technical-military expressions, modifies organically absolute and relative relations in the international field too". Tradução própria.

${ }^{14}$ COX, Robert W. ob. cit, p. 59. "Those states which are powerful are precisely those which have undergone a profound social and economic revolution and have most fully worked out the consequences of this revolution in the form of state and of social relations". Tradução própria.

${ }^{15}$ Idem, pg. 62. "It is also a complex of international social relationships which connect the social classes of the different countries. World hegemony is describable as a social structure, an economic structure, and a political structure; and it cannot be simply one of these things but must be all three. World hegemony, furthermore, is expressed in universal norms, institutions and mechanisms which lay down general rules of behaviour for states and for those forces of civil society that act across national boundaries". Tradução própria.
} 
A partir desta leitura do conceito é que o mesmo Robert Cox afirma serem as organizações internacionais um dos principais mecanismos de consolidação da hegemonia internacional. Elas desempenham este papel por: incorporarem as regras que facilitam a expansão da ordem hegemônica mundial; serem, elas mesmas, o produto da ordem hegemônica mundial; legitimarem, ideologicamente, as normas/ valores da ordem mundial; cooptarem as elites dos países periféricos e absorver idéias contra hegemônicas ${ }^{16}$.

\section{A supremacia americana e a lógica do desarmamento}

O escritor americano, Joseph Nye, descreve a supremacia dos EUA como segue:

A supremacia dos EUA estende hoje à economia, à moeda corrente, às áreas militares, ao estilo de vida, à língua e aos produtos da cultura popular que inunda o mundo, formando pensamento e fascinando mesmo os inimigos dos EUA. Em poderio militar, nós (os EUA) somos a única nação dotada tanto de armas nucleares como de forças convencionais de alcance global. Economicamente respondemos por $27 \%$ da produção mundial, o equivalente (a preços de mercado) à soma da participação dos outros três países mais ricos (o Japão, a Alemanha e a França). (...) O comércio americano é o triplo do europeu, e sediamos sete dos dez maiores vendedores de software. (...) Quanto ao poder brando, os EUA são, de longe, o maior exportador de filmes e programas de televisão do mundo (...). Os EUA são o modelo econômico dominante para o resto do mundo desenvolvido e em desenvolvimento ${ }^{17}$.

Para preservar essa supremacia na ordem mundial, os EUA adotaram uma política agressiva, principalmente a partir da segunda guerra mundial. Envolvimento direto em conflitos armados, patrocínio de grupos de oposição a regimes contrários aos seus interesses e intervenção em questões internacionais que não conheceram limites no próprio direito internacional, foram algumas das vias utilizadas sem qualquer parcimônia ${ }^{18}$.

\footnotetext{
${ }^{16}$ Ibidem.

${ }^{17}$ NYE, Joseph S. The Paradox of American Power: why the world's only superpower can't go it alone. United States: Oxford University Press, 2002, p. 36. "U.S. supremacy today extends to the economy, currency, military areas, lifestyle, language and the products of mass culture that inundate the world, forming thought and fascinating even the enemies of the U.S. In military power, we (US) are the only country with both nuclear weapons and conventional forces with global reach. Economically we have a 27 percent share of world product which (at market prices) was equal to that of the next three countries combined (Japan, Germany, France). We are the home of fifty-nine of the hundred largest companies in the world by market value (compared to thirty-one for Europe and seven for Japan). In direct foreign investment, we invested and received nearly twice as much as the next ranking country (Britain). American e-commerce was three times that of Europe, and we are the home of seven of the top ten software vendors. Forty-two of the top seventyfive brands were American, as well as nine of the top ten business schools. In terms of soft power, the US is far and away the number one film and television exporter in the world, although India's "Bollywood" actually produces more movies per year. We also attract the most foreign students each year to our institutions of higher education, followed by Britain and Australia. In addition to students, over 500,000 foreign scholars were in residence at American educational institutions in 2000. The US is the dominant economic model for the rest of the developed world and much of the developing world". Tradução própria.
}

${ }^{18}$ CHOMSKY, Noam. O que o Tio Sam realmente quer. Tradução de Sistílio Testa e Mariuchka Santarrita. Brasília: Editora Universidade Brasília, 1999, p. 9-36. 
Apesar de tudo isso, os EUA não trataram de buscar a preservação de seu domínio apenas a partir do uso da força: "por meio de uma bem urdida estratégia de disseminação de seus valores, acabaram transformando o sonho americano no sonho de muitos ao redor do mundo, com isso quebrando resistências culturais à sua presença; são por demais evidentes as influências do modo de vida americano (american way of life) sobre outras culturas - em especial às dos países do chamado terceiro mundo, para merecerem aqui maiores explicitações"19. Isto demonstra que "os EUA foram nações desenvolvidas, que transpuseram as fronteiras nacionais para se tornarem fenômenos de expansão internacional" 20 " OO conceito hegemônico de ordem mundial é edificado não somente sob a regulação dos conflitos entre Estados, mas também sob uma sociedade civil compreendida globalmente"21.

Transportando estas idéias para a cena de segurança internacional, se faz necessário, antes de tratar das ações estadunidenses para disseminação das armas nucleares, lembrar sucintamente o desarmamento.

No início das discussões sobre desarmamento, este era aceito por uma potência por imposição ou por perder uma guerra. Mas depois de alguns incidentes nucleares e da lúgubre experiência sofrida por Hiroshima e Nagasaki, o desarmamento se tornou uma questão defendida por um notável número de países. "A maioria da comunidade internacional aos poucos parece ter chegado à conclusão de que a posse de armamento nuclear não seria a melhor garantia para sua segurança"22. Contudo, o desarmamento multilateral tem sido em geral impossível de ser obtido. Existem quatro motivos descritos por Wight ${ }^{23}$ para essa impossibilidade. O primeiro motivo ele chama de egoísmo nacional. "Toda potência em geral considera melhor para sua segurança que outras potências se desarmem enquanto ela própria permanece livre para decidir o nível de suas armas"24. $\mathrm{O}$ segundo motivo é a dificuldade de se comparar potências. "Toda potência tem de se subordinar o desarmamento à segurança, e não pode discutir o desarmamento sem possuir uma idéia suficientemente clara daquilo que poderá ter de defender no futuro" 25 . O terceiro motivo é que o fato de limitar ou reduzir os armamentos tenderia a consolidar o status quo e a petrificar o equilíbrio do poder. "Um acordo para redução de armas, portanto, só é provável se as potências em questão estiverem dispostas a aceitar tacitamente a distribuição existente de território e de recursos, ou se os descontentes puderem ser coagidos" ${ }^{26}$. O quarto e último motivo é que "se o equilíbrio de poder for artificial, no sentido de que as

\footnotetext{
${ }^{19}$ MICHELS, Op. Cit, p. 132.

${ }^{20}$ COX, Robert W. ob. cit, p. 59. "The U.S. were nation-based developments which spilled over national boundaries to become internationally expansive phenomena". Tradução própria.

${ }^{21}$ Idem, p. 61. "The hegemonic concept of world order is founded not only upon the regulation of inter-state conflict but also upon a globally-conceived civil society”. Tradução própria.

${ }^{22}$ DUARTE, Sergio. "A sétima conferência de exame do Tratado de Não-Proliferação de Armas Nucleares uma avaliação". In Política Externa. A democracia na América Latina. Vol. 14 No 4 março/ abril/ maio 2006, p. 120.

${ }^{23}$ WIGHT, Martin. A política do poder. Tradução de Carlos Sérgio Duarte (2a edição). Brasília: Editora Universidade de Brasília, Instituto de Pesquisa de Relações Internacionais; São Paulo: Imprensa Oficial do Estado de São Paulo, 2002, p. 269-290. Durante todo o capítulo o autor relata as guerras e acordos internacionais envolvendo a questão do desarmamento. Desta forma é possível verificar por que os países desejam a efetiva realização do desarmamento.

${ }^{24}$ Idem, p. 283.

${ }^{25}$ Idem, p. 284.

${ }^{26}$ Idem, p. 287.
} 
armas não correspondem ao potencial de guerra, as potências satisfeitas podem declarar que são incapazes de desarmar sem terem garantias para sua segurança"27.

Desta forma pode-se observar a dificuldade de se conseguir um desarmamento multilateral. E pelos mesmos motivos, todos os países desejam o desarmamento do país vizinho. O mesmo não é diferente com os EUA. Ao longo dos anos, eles têm se batido empenhadamente para que o desarmamento efetivamente ocorra em todos os países, menos no seu.

As cinco principais iniciativas de não-proliferação por parte dos EUA desde 1945 foram: o Plano Baruch, a Iniciativa Átomos para a Paz (Atoms for Peace); o TNP de 1968; regimes de controle de tecnologias de proliferação e Iniciativa de Contraproliferação de Defesa.

Em 13 de junho de 1946, Bernard Baruch apresentou à Comissão das Nações Unidas de Energia Atômica (United Nations Atomic Energy Commission - UNAEC) o plano que carregou seu nome. Ele propôs a criação de uma Autoridade Internacional de Desenvolvimento Atômico (International Atomic Development Authority - IADA) que seria incumbida do gerenciamento total de toda atividade de energia atômica que fosse potencialmente perigosa para a segurança mundial. A proposta era controlar as reservas de minerais radioativos, ao redor do mundo, propondo que todos os países com reservas de minerais nucleares devessem colocar os seus suprimentos sob a responsabilidade da IADA. Esta proposta não logrou aprovação por ter sido rejeitada pela União Soviética.

Três anos mais tarde, em 1949, a União Soviética inaugurou o seu primeiro engenho nuclear e Washington receou um ataque nuclear contra os EUA. A questão era saber quando. Baseado no receio de que o crescente poderio nuclear soviético punha em risco toda a base de mobilização industrial e militar americana, a administração Eisenhower desenvolveu o programa Átomos para a Paz (1953). Este programa foi um discurso feito pelo presidente dos Estados Unidos da América, Dwight David Eisenhower, na Assembléia Geral das Nações Unidas, em oito de dezembro de 1953. Quatro anos depois, em 29 de julho de 1957, surgiu a AIEA, com o estatuto aprovado por 81 nações definindo seus pilares de trabalho: segurança nuclear, salvaguardas e transferência de tecnologias e tendo um papel fundamental na preservação dos usos pacíficos da energia nuclear para evitar a proliferação de armamentos nucleares ${ }^{28}$.

O domínio da tecnologia nuclear por mais países e a obtenção de armas nucleares pela França, em 1960, e China, em 1964, preocupou a sociedade mundial da difusão desse conhecimento e das suas possíveis conseqüências. Portanto, com o intuito de frear a propagação de armas nucleares e trabalhar em direção a uma eventual eliminação das mesmas, foi aprovado em 1968 o TNP. Este estabeleceu quais eram os cinco países possuidores de armas nucleares (EUA, União Soviética, Reino Unido, França e China), enquanto os demais, ao assinarem o Tratado, não poderiam produzi-las e também, junto à AIEA, deveriam assinar um acordo de salvaguardas sobre seus materiais nucleares ${ }^{29}$. Desta forma, Silva vê este Tratado como "assimétrico e discriminatório por dispensar a inspeção em países nuclearmente armados e inspecionar somente os desarmados" ${ }^{130}$.

É neste contexto que os EUA e mais alguns países fornecedores de tecnologia nuclear (Reino Unido, França, Alemanha, Canadá e Japão), após a conclusão do TNP,

\footnotetext{
${ }^{27}$ Idem, p. 289.

${ }^{28}$ FISCHER, David. History of the International Atomic Energy Agency : the first forty years / by David Fischer. Vienna: The Agency, 1997, p. 1. Disponível em: 〈http://wwwpub.iaea.org/MTCD/publications/PDF/Pub1032_web.pdf>. Acesso em: 01 de junho de 2006.

${ }^{29}$ Idem, p. 2.

${ }^{30}$ Da SILVA, Othon. L. P. "As inspeções Nucleares no Brasil e o Mictório Público Francês". Economia e Energia. Ano VIII. Nº 44. Maio-Junho 2004. Ele é engenheiro naval, mecânico e nuclear.
} 
começaram a desenvolver negociações para definir formas de restringir a transferência de tecnologia, materiais e equipamento para países em zonas de instabilidade. É sob esta perspectiva que foi criado em 1974 o Grupo de Países Fornecedores de Combustível Nuclear (Nuclear Suppliers Group (NSG)) que definiu orientações para a transferência de tecnologia nuclear (The Guidelines for Nuclear Transfer). Mais tarde, em 1987, foi criado o Grupo de Controle Tecnológico de Mísseis (Missile Technology Control Group) restringindo a transferência deste tipo de tecnologia apenas para projetos de lançamentos de satélites militares, aéreos ou mísseis de curto alcance. Faltavam, porém, mecanismos para impor as normas estabelecidas. Em 1974, a Índia realizou, à margem do TNP, o seu primeiro teste nuclear e desta forma ficou comprovado que esse teste só foi possível graças a um regime muito brando de restrições americanas à transferência das tecnologias aplicáveis. Novas medidas foram tomadas, mas mesmo assim, uma década mais tarde, na Guerra Irã-Iraque de 1980/1988, quando o Iraque utilizou armas químicas contra o Irã, constatou-se que não foi possível impedir a construção de um avançado míssil balístico.

Confrontados com o insucesso das políticas de não-proliferação, os EUA desenvolveram a Iniciativa de Contraproliferação de Defesa (Counterproliferation Initiative), esta foi apresentada pela primeira vez em 7 de Dezembro de 1993, por Lee Aspin, então secretário da Defesa da Administração Clinton, sob a idéia de que não lhes restava outra hipótese senão desenvolver capacidades militares para neutralizar essa ameaça. Não foi, porém, esta opção que prevaleceu na crise de 1992 com a Coréia do Norte, na qual os EUA preferiram negociar o Agreed Framework de 1994, que dava à Coréia do Norte compensações significativas pela desistência de prosseguir o seu programa nuclear. Como se sabe, este acordo acabou também por não funcionar e tudo voltou ao princípio em 2002, demonstrando mais uma vez as limitações dos atuais mecanismos de combate à proliferação de armas nucleares ${ }^{31}$.

Em um importante documento de 1995, o comando estratégico americano (STRATCOM) descreveu as armas nucleares como as mais valiosas do arsenal, pois diferentemente do que ocorre com as armas químicas ou biológicas, a destruição extrema de uma explosão nuclear é imediata, com poucos - se é que existem - paliativos para reduzir seus efeitos. Além disso, as armas nucleares são sempre capazes de lançar uma sombra sobre quaisquer crises ou conflitos, devendo ser visíveis e estar a postos ${ }^{32}$.

De fato, nem os tratados internacionais nem a atividade da AIEA estão sendo eficazes em impedir a decisão de um Estado vir a possuir um arsenal nuclear. Mas isso não impediu esta organização internacional de tentar fechar o cerco quanto às salvaguardas.

\section{O Protocolo Adicional e a renovação do velho.}

Para melhorar as regras de salvaguardas, a AIEA propôs em 1997 o Protocolo Adicional $^{33}$. Este surgiu por ter se encontrado no Iraque, na ocasião da Guerra do Golfo, indícios de que este país não estava cumprindo com suas obrigações junto ao TNP e a AIEA. A proposta foi feita pela comissão de diretores composta por 35 membros $^{34}$, sendo

\footnotetext{
${ }^{31}<$ http://www.jornaldefesa.com.pt/conteudos/view txt.asp?id=13 $>$ acesso em 7 de julho de 2006.

${ }^{32}$ CHOMSKY, Noam. O Império Americano: hegemonia ou sobrevivência. Tradução de Regina Lyra. Rio de Janeiro: Elsevier, 2004, p. 221.

${ }^{33}$ O nome completo é: Protocolo Adicional Para o Acordo entre Estados e a Agência Internacional de Energia Atômica para a Aplicação de Salvaguardas.

${ }^{34}$ Ver <http://f40.iaea.org/worldatom/About/Board/bg1997.html > Acesso em 16 de junho de 2006. The 35 Member States on the Board for 1997-98 are Argentina, Australia, Belgium, Brazil, Canada, China, Colombia, Cuba, Czech Republic, France, Germany, Ghana, India, Italy, Hungary, Japan, Republic of Korea, Malaysia, Mexico, Morocco, Namibia, Netherlands, New Zealand, Pakistan, Peru, Portugal, Russian
} 
um deles os EUA. Desde então, ficou aprovado um modelo, os quais os países deveriam seguir para realizar o seu acordo. Lembrando que este modelo deve ser seguido por todos os países que são signatários do TNP e que não possuem armas nucleares, já que para os países nuclearmente armados o Protocolo é feito da forma como eles quiserem, com as cláusulas que mais lhe convierem. Os $\mathrm{EUA}^{35}$, como forma de exemplo, assinaram este Protocolo, mas ainda não ratificou o que significa que ele não tem nenhuma consequiência para o país em questão. Contudo, eles defendem a assinatura e a ratificação deste Protocolo por todos os países. Desta forma, eles ganham mais um forte instrumento para seu grande objetivo, que é restringir a proliferação de armas nucleares por meio da $\mathrm{AIEA}^{36}$.

A AIEA inspeciona aquilo que é declarado pelos países, e com este Protocolo os Estados se obrigam a detalhar mais essas declarações ${ }^{37}$. Com ele, a AIEA ganha mais poderes como: inspeções mais intensas, acesso irrestrito e sem aviso prévio. $\mathrm{O}$ compromisso principal é conferir a ausência de atividades e material nuclear em locais declarados a fim de determinar que o programa nuclear declarado é consistente com o planejado; as atividades nucleares e os tipos de material nuclear em locais declarados são consistentes com aqueles declarados; os inventários e fluxos de materiais nucleares, a produção e as importações e exportações correspondem à utilização prevista no programa nuclear declarado; a manufatura e importação de equipamentos especificados e materiais não nucleares são compatíveis com o programa nuclear declarado; a situação de instalações fechadas está em conformidade com a declaração do país; as atividades de pesquisa e desenvolvimento relacionadas com o ciclo do combustível estão de acordo com os planos declarados sobre futuros desenvolvimentos do programa nuclear; e os esclarecimentos fornecidos pelo Estado resolvem qualquer questão ou inconsistência relativa às informações prestadas e àquelas disponíveis pela AIEA, incluindo informações sobre atividades desenvolvidas no passado.

No que se refere à segurança dos EUA pela implementação do Protocolo é preciso perceber que para eles, quanto maior o número de países que dispõem de armas nucleares, maior o risco de que elas sejam deliberadamente utilizadas não para dissuadir, mas para aniquilar, ou que um conflito seja desencadeado por engano, ou que um país bombardeie preventivamente as instalações de seus adversários, ou ainda que armas ou matérias físseis caiam nas mãos de grupos criminosos. E quanto mais países tiverem armas nucleares pior será para os EUA, pois os Estados ganharão mais força, dificultando qualquer tipo de influência que os EUA possam querer exercer sobre eles. Haverá uma perda relativa do poderio militar estadunidense. Isso nada mais é do que uma continuidade da política externa norte-americana. A aposta de não-proliferação enquanto assegura seu poder de continuar possuindo armas nucleares, nada tem de novo; ela é, simplesmente, parte do processo de contínua renovação do velho.

Federation, Slovenia, South Africa, Sweden, Tunisia, United Arab Emirates, United Kingdom of Great Britain and Northern Ireland, United States of America, and Vietnam. The Chairman of the Board of Governors is Ambassador Yuji Ikeda of Japan.

${ }^{35}$ Eles alegam que assinaram o tratado para dar exemplo aos outros países. Ou seja, ganham legitimidade para pressionar outros países a fazerem o mesmo. Ver <http://www.energy.gov/news/1880.htm> Acesso em $12 \mathrm{de}$ julho de 2006.

36 Para entender a influência que os Estados Unidos exercem sobre a AIEA, ver http://www.iea.usp.br/iea/revista/online/eua/bustani.pdf acesso em 11 de julho de 2006.

${ }^{37}$ No artigo 2 do Protocolo Adicional os Estados ficam obrigados a declarar à AIEA informações como localização onde possa estar ocorrendo desenvolvimento de atividades nucleares; descrição de cada construção no site e sua utilidade; localização, status operacional e produção estimada anual para cada mina de urânio e plantas de concentração; detalhes sobre o material utilizado; lista de todos os equipamentos utilizados no serviço nuclear e também as pessoas que lá trabalham. E o Estado deve fornecer à AIEA qualquer informação adicional que ela desejar. 


\section{Hegemonia estadunidense e Hegemonia gramsciana}

Gramsci escreveu que não é preciso a classe hegemônica assumir o comando, desde que os valores hegemônicos já estejam infiltrados na classe social. Transportando isso para o cenário internacional é possível verificar que a política de não-proliferação nuclear é um tema que desponta uma quase unanimidade de opiniões. Os 187 países que assinaram o tratado do TNP (todos os países do mundo, menos quatro), parecem concordar com a hipótese de um mundo sem mais armas nucleares. A AIEA, para manter essa afirmativa funcionando, conta com a comissão de diretores, que é um grupo de países que se reúnem anualmente para discutir e votar quais serão as melhores atitudes a serem tomadas para o cumprimento dos tratados internacionais sobre assuntos nucleares. Para ter uma devida ordem nestas reuniões, cada ano um Estado é eleito para presidi-la. Como os EUA sempre estiveram empenhados nessas discussões, encabeçando muitos projetos internacionais, imagina-se que a cadeira de presidente da comissão de diretores da AIEA fosse um grande alvo para este país. Contudo, como nos mostra o conhecimento gramsciano, os EUA apesar de sempre ter feito parte desta comissão a presidiu poucas vezes. Contudo, é importante ressaltar que o primeiro diretor geral da AIEA foi o norte americano Sterling Cole, presidindo a agência nos seus primeiros anos de formação, de 1957-1961.

Todavia, uma das formas que o país consegue a condição hegemônica é através das Organizações Internacionais, segundo Robert Cox. Apesar de os EUA não estarem à frente da AIEA (o que para Gramsci não é preciso), essa organização incorpora, legitima os valores da ordem mundial. Pois, por meio de sua história, observa-se que há um intenso trabalho na não-proliferação de armas nucleares, o que é um desejo das potências nucleares, que outros países não adquiram o poder de fabricar armas nucleares, enquanto a política de desarmamento não é um assunto discutido com tanto afinco.

Uma ação prática de desarmamento foi mostrada quando os EUA e a Federação Russa, com o acordo de Moscou de maio de 2002, concordaram em limitar a 1700-2200 as ogivas nucleares ativas. De fato isso ocorreu. Mas foram materiais velhos, antigos, gastos, com uma tecnologia já ultrapassada, e que seriam destruídos de qualquer jeito, não importando se isso seria bem visto pela comunidade internacional. Como foi relatado pelo diplomata Cavalcanti ${ }^{38}$. A estratégia foi muito bem montada, agora essa atitude é vista como algo nobre que esses países fizeram.

No entanto, para analisar a questão dos EUA frente ao Protocolo Adicional é necessário observar sua atitude para com este Protocolo. De acordo com o sítio oficial de energia dos EUA, eles são os principais defensores da implementação do regime de nãoproliferação com a total adoção do Protocolo Adicional ${ }^{39}$. Todavia, a demora da assinatura por parte de alguns países faz com que os EUA comecem a pensar em maneiras coercitivas para que isto aconteça. Como, por exemplo, fazer desse Protocolo um requisito básico para o comércio de materiais nucleares ${ }^{40}$. A medida diplomática, por meio dos modos legais do direito internacional para a aprovação do Protocolo demonstra o consentimento, o momento do consenso, que Coutinho lembra ser o que significa hegemonia. Porém, a coerção ideológica também deve estar presente para obter-se uma hegemonia completa, conforme Gramsci a idealizava. E é exatamente isso o que existe neste Protocolo.

\footnotetext{
${ }^{38}$ Entrevista realizada dia 12 de julho de 2006, com o assessor da área nuclear da Divisão de Desarmamento e Tecnologias Sensíveis do Ministério das Relações Exteriores, Ricardo Henrique Silva de Sá Cavalcanti.

${ }^{39}$ Ver http://www.energy.gov/news/1768.htm Acesso em 20 de julho de 2006. E http://www.energy.gov/news/1809.htm

${ }^{40}$ Ver http://www.nnsa.doe.gov/docs/speeches/2005/speech IAEA Nuclear Security final presentation.pdf
} 


\section{Considerações Finais}

Como se viu ao longo deste artigo, o pensamento de Antonio Gramsci tem um papel inovador. Apesar de suas análises terem sido feitas para explicar as relações dentro do Estado italiano, faz-se possível utilizá-las para explicar as complexas relações existentes nas relações internacionais. Na medida em que suas categorias permitem uma investigação ampla dos vários aspectos da sociedade internacional, torna possível a elaboração de um panorama mais completo de uma dada realidade. O conceito central é o de hegemonia.

O sentido dado de hegemonia, como forma de imperialismo, pelo senso comum, diverge daquele utilizado por Gramsci. Ele defendia que a hegemonia é a supremacia de um grupo ou classe sobre outras classes ou grupos, por meio do consentimento. Isto aponta que para Gramsci o processo de hegemonia representa a primazia da sociedade civil sobre a sociedade política, por meio da produção do consenso. Ou seja, a hegemonia é à busca do consenso no âmbito da sociedade civil. Esta foi uma das maiores contribuições de Gramsci para a teoria política.

Não obstante, o esforço de adaptar o pensamento e os mecanismos de hegemonia gramscianas para a análise do atual cenário internacional pode trazer agradáveis resultados e revelar-se uma boa ferramenta para se compreender as ações da hegemonia mundial nos dias atuais.

No que respeita especificamente a atitude dos EUA frente ao Protocolo Adicional, percebe-se, à luz do ideário gramsciano, que os EUA têm agido de forma a criar uma cultura internacional da necessidade da aprovação, assinatura, ratificação e cumprimento deste Protocolo. E pode-se a afirmar que este objetivo tem sido alcançado por meio do mecanismo de hegemonia, através dos quais as normas universais de uma hegemonia mundial são expressas, e que é um instrumento de disseminação de ideologia de interesse dos norte-americanos, a AIEA.

Já se tem hoje a aprovação, para o Protocolo Adicional, de 114 países, sendo que destes 109 assinaram e para somente 76 países ele entrou em vigor ${ }^{41}$. Isto evidencia uma aprovação de $62 \%$ dos países que assinaram o TNP, em nove anos de trabalho. Assim sendo, a veemente defesa dos EUA para com o Protocolo Adicional tem sido uma tentativa de preservação de sua hegemonia mundial, desde que não mude sua estratégia de implementação deste Protocolo.

\section{Referências Bibliográficas}

ABRAHAM, Spencer. Council on Foreign Relations. Remarks Prepared for Energy Secretary Abraham. January 13, 2005. <http://www.energy.gov/news/1880.htm> Acesso em 12 de julho de 2006.

ABRAHAM, Spencer. International Atomic Energy Agency. Remarks Prepared for Energy Secretary Abraham. September 20, 2004. <http://www.energy.gov/news/1809.htm> Acesso em 20 de julho de 2006

ABRAHAM, Spencer. United Nations, New York, NY. Remarks by Secretary of Energy Spencer Abraham. November 5, 2003. 〈http://www.energy.gov/news/1768.htm> Acesso em 20 de julho de 2006

\footnotetext{
${ }^{41}$ SAFEGUARDS AND VERIFICATION. < http://www.iaea.org/OurWork/SV/Safeguards/sg protocol.html> Acesso em 14 de agosto de 2006.
} 
ALVEM, Carlos Feu. O Brasil e o Protocolo Adicional ao acordo de Salvaguardas. Disponível em: 〈http://ecen.com/eee43/eee43p/protocolo_adc.htm>

BROOKS, Linton F. Preventing Nuclear Terrorism: Towards an Integrative Approach. IAEA International Conference on Nuclear Security. March 16, 2005. London, England. <http://www.nnsa.doe.gov/docs/speeches/2005/speech IAEA Nuclear Security final pres entation.pdf $>$ Acesso em 12 de julho de 2006.

BUSTANI, José Maurício. Em defesa do programa nuclear brasileiro. Artigo do embaixador do Brasil em Londres. Folha de São Paulo: 11/10/2004. Disponível em: <http://www.itamaraty.gov.br/portugues/politica externa/discursos/discurso detalhe.asp?I D_DISCURSO=2476>

BUSTANI, José Maurício. O Brasil e a OPAQ: diplomacia e defesa do sistema multilateral sob ataque. 〈http://www.iea.usp.br/iea/revista/online/eua/bustani.pdf $>$ acesso em $11 \mathrm{de}$ julho de 2006.

CARNEVALI, Giorgio. "A Teoria da política Internacional de Gramsci". In MEZZAROBA, Oribes (org.). Gramsci, Estado e Relações Internacionais. Florianópolis: Fundação Boiteux, 2005.

CHOMSKY, Noam. O que o Tio Sam realmente quer. Tradução de Sistílio Testa e Mariuchka Santarrita. Brasília: Editora Universidade Brasília, 1999.

COUTINHO, Carlos Nelson. Gramsci: um estudo sobre seu pensamento politico. Rio de Janeiro: Civilização Brasileira, 1999.

COX, Robert W. "Gramsci, Hegemony and International Relations: an essay in Method" In GILL, Stephen (org.). Gramsci, Historical Materialism and International Relations. Cambrigde, Reino Unido: Cambrigde University Press, 1993.

Da SILVA, Othon. L. P. "As inspeções Nucleares no Brasil e o Mictório Público Francês". Economia e Energia. Ano VIII. No 44. Maio-Junho 2004.

DUARTE, Sergio. "A sétima conferência de exame do Tratado de Não-Proliferação de Armas Nucleares - uma avaliação". In Política Externa. A democracia na América Latina. Vol. $14 \mathrm{~N}^{\circ} 4$ março/ abril/ maio 2006.

FISCHER, David. History of the International Atomic Energy Agency : the first forty years / by David Fischer. Vienna: The Agency, 1997, p. 1. Disponível em: <http://wwwpub.iaea.org/MTCD/publications/PDF/Pub1032_web.pdf>. Acesso em: 01 de junho de 2006.

GILL, Stephen. "Gramsci and Global Politics: towards a post-hegemonic research agenda" In GILL, Stephen (org.). Gramsci, Historical Materialism and International Relations. Cambrigde, Reino Unido: Cambrigde University Press, 1993.

LAMAZIERE, Georges. Ordem, Hegemonia e Transgressão. Brasília: Funag, 1998. Curso de Altos Estudos do Instituto Rio Branco.

MEZZAROBA, Oribes. "Gramsci e a Hegemonia" In MEZZAROBA, Oribes (org.). Gramsci, Estado e Relações Internacionais. Florianópolis: Fundação Boiteux, 2005.

MICHELS, Gilson Wessler. "Guerra do Iraque e Hegemonia norte-americana: Uma leitura a partir de Gramsci" In MEZZAROBA, Orides (org.). Gramsci: Estado e Relações Internacionais. Florianópolis: Fundação Boiteux, 2005. 
MODELO DE PROTOCOLO ADICIONAL para o acordo entre Estado e a Agência Internacional de Energia Atômica para a Aplicação de Salvaguardas. Model Protocol Additional To The Agreement(S) Between State(S) And The International Atomic Energy Agency For The Application Of Safeguards. <http://www.iaea.org/Publications/Documents/Infcircs/1998/infcirc540corrected.pdf>

NYE, Joseph S. The Paradox of American Power: why the world's only superpower can't go it alone. United States: Oxford University Press, 2002.

PORTELLI, Hugues. Gramsci e Bloco Histórico. Rio de Janeiro: Paz e Terra, 1977. Cap. 1

SEMERARO, Giovanni. Gramsci e Sociedade Civil. Rio de Janeiro: Vozes, 1999. Cap. 2

WIGHT, Martin. A política do poder. Tradução de Carlos Sérgio Duarte (2 edição). Brasília: Editora Universidade de Brasília, Instituito de Pesquisa de Relações Internacionais; São Paulo: Imprensa Oficial do Estado de São Paulo, 2002. 